\title{
COMMUNICATING IN THE VERNACULAR: DIGITAL TYPE AND DESIGN IN TAMIL CINEMA ADVERTISING
}

\author{
Margaret Thomas \\ Stella Maris College
}

Email: thomasmargaret1508@gmail.com

\begin{abstract}
Typography is defined as 'the arrangement and appearance of printed matter'. However, in today's context it can be updated to include the design arrangement of any communicative material. 'Grammatology' according to Jacques Derrida, is the study of writing as a distinctive form of representation and within its domain are the material forms and processes of typography and design. Typography, in relation to structuralist theory, as instituted by type designers Bodoni and Didot charts a shift from the humanist understanding of printed letters as reflections of handwritten marks or classical proportions towards the modernist view of typography as the endless manipulation of abstract elements. It is possible to draw parallels with modern English typography and similar stylistic developments in the title designs for Tamil cinema posters and hoardings where words, images, and objects participate in a culturally determined meaning characterizing a verbal language. Though cinema in India has been in existence for over a hundred years, in recent years there has been an explosion of new and highly inventive typefaces. This paper will examine how digital typography and design as a vernacular cultural force sought to reach a varied audience in the titles created for Tamil cinema publicity from the year 2000 onwards. The designer developed visual codes that forced the audience to reevaluate conventional Tamil typeface design. While earlier Tamil cinema advertising used handcrafted type and imagery to promote the film, it changed with digital technology enabling the typographic design of the titles of the films to indicate the genre be it mythological, historical, patriotic, romantic or action oriented.
\end{abstract}

Keywords: Typography, Design, Vernacular, Visual Code

The hybrid nature of Indian cinema is a result of a new public culture that arose during the nineteenth century, bringing together traditional Indian images with industrial technology made available by the English colonizer. An integral part of Indian culture and mass psyche, cinema is an economic and cultural institution, made and watched in a commercial context. The growth of Indian film production and the significance of movies in Indian society since Independence in 1947 have been closely associated with the rapid expansion of Indian cities and of urbanism as a lifestyle, which has taken place concurrently. Chennai, formerly Madras, is regarded as the cultural capital of southern India and has one of the most active music, dance, theatre and art scenes. Its film industry is the second largest in the country. Till about 1960, Madras was the centre of all South Indian language cinema productions, the largest segment of which was made up of Tamil films. To reach its targeted consumer groups, Tamil cinema as a product needs to be advertised, just as every other brand, product or service. As technology developed so did the publicity for Tamil cinema; it evolved from hand-rendered typography posters to a combination of image and type made possible with newer printing techniques.

While much has been written about the visual culture of Mumbai-based Bollywood cinema, as reflected in its dynamic advertising, such as posters, hand-rendered hoardings and so on, little has been published on Tamil film promotionals. Visual culture in Chennai's urban space has been extensively shared by Tamil cinema hoardings and posters. This almost undocumented area of academic study—outdoor advertising for Tamil films in Chennai during the digital era - forms the area of my research. In addition to promoting films, advertising for 
Tamil cinema employed the visual vocabulary of the period to reflect the ideas, beliefs, attitudes and values of the cultural environment. In other words, it is representative of its times. The objective of this paper is to highlight the new aesthetic of graphic design, particularly typography in Tamil cinema publicity as dictated by digital technology. The examples cited are geographically limited to the city of Chennai, and are investigated using relevant theories and concepts.

While Tamil cinema itself is a significant medium of communication, its advertising in Chennai has equally been recognized as an influential visual art form that has captured the attention of the millions who inhabit the city. With the swift development of technology, advertising for Tamil cinema has grown much beyond the twodimensional format. Digital technology was exploited by the Tamil film industry for its promotionals, particularly posters and hoardings, from the year 2000 onwards. Posters and handbills were used for Tamil cinema publicity ever since mechanical printing came to India in the early nineteenth century, and particularly to Sivakasi in Tamil Nadu around the 1950s. It was also around the 1950s that hoardings or 'banners' and 'cutouts', as they were referred to at that time, came to be employed for advertising Tamil cinema in Chennai. For the posters, the designs were manually rendered as art works, and mechanically printed for mass distribution, whereas all banners and cut-outs were hand-rendered by skilled artists who were apprenticed to banner companies. Once completed, the banners were stretched at the site by laborers employed by the banner companies. Affordability of digital technology in the twenty-first century displaced hand-painted banners. With the coming of digital technology not only was the skilled artist displaced, but there was a complete overhaul in the design of film publicity lending it an aura of sophistication and modernity not tapped thus far, evidently because of the limitation of the mediums employed. The designers and photographers from the advertising industry in Chennai played a major role in upgrading Tamil cinema publicity assisted by the advanced technology made available to them.

The paper will focus on the impact of digital technology on Tamil cinema publicity, moving the visual art form from the realm of commercial art to graphic design. Examples that are cited in the paper are of posters and hoardings that starkly differ from traditional film advertising that was prevalent until the introduction of digital technology in terms of visuals, design, layout, typography, and so on.

Walter Benjamin believed that the concept of 'progress' and the concept of 'period of decline' are two sides of one and the same thing (Benjamin, 1936). In this case, the belief is linked to the immense significance of recent developments in technology, or in other words, it forecast a kind of technological determinism (Lister, 1995). 'Technological determinism,' a term coined by social scientist Thorstein Veblen in the 1920s, refers to the idea that technology is the agent of social change. The concept was forged as a social attitude in eighteenth century Britain, in which progress was measured in industrial terms: speed of movement, volume of production, and so on (Murphie and Potts, 2003). However, it is still relevant today as reflected in 'information society' or 'computer age' - terms that are increasingly in circulation in the present age. Chennai betrays the technological determinist notion that society is shaped by its dominant technologies and is influenced by it, as is repeatedly evident in the advertisements that reflect the latest technologies on different surfaces in the city.

With the advent of digital technology and arrival of the computer in the field of design, not only does type and imagery offer an integrated and seamless graphic solution, but is also cost effective, changing the very nature of design. From early 1990s there has been an explosion of new and highly inventive typefaces in India. Digital typography and design as a vernacular cultural force sought to reach a varied audience. Using visual codes that were dramatic, designers reinvented conventional Tamil typeface to create stunning titles for their cinema publicity. From the 1960s to late 1990s Tamil cinema posters and hoardings used handcrafted type and imagery which was labour intensive to promote the film. All this changed in the late 1990s with digital technology. This paper will focus, specifically on the refreshingly modernist approach to digital typography in title designs for Tamil cinema employed by specialists. Innovative designs not visible in mainstream Tamil cinema advertising thus far have been the criteria for selection of specific images cited in the paper. 
The Oxford English Dictionary defines typography as 'the arrangement and appearance of printed matter'. However, in today's context it can be updated to include the design arrangement of any communicative material ranging from posters, hoardings, TV screen idents, signages and so on. Jacques Derrida, who introduced the theory of deconstruction in the mid 1980s, used the term 'grammatology' to name the study of writing as a distinctive form of representation. Falling within the domain of grammatology are the material forms and processes of typography and design. If writing is a copy of spoken language, typography is a mode of representation even farther removed from the primal source of meaning in the mind of the author. The art of typography includes the design of letterforms for reproduction and the arrangement of characters into lines of text. Typographic features include the choice of typefaces; the spacing of letters, words, lines and columns; and the pattern formed by these graphic distinctions across the body of a document (Lupton and Miller, 1996). Structuralist typography inaugurated by Giambattista Bodoni of Italian origin and French type-founder Francois Ambroise Didot is continued by designers of advertising display faces. By shifting the emphasis from the individual letter to the overall series of characters, structuralist typography exchanged the fixed identity of the letter for the relational system of the font. Designers have continued to invent typefaces that manipulate the formal system of the alphabet and attempt to defamiliarize the experience of reading.

It is possible to draw parallels with the history of modern English typography and similar stylistic developments in the title designs for Tamil cinema. Typography, charts a shift from the humanist understanding of printed letters as reflections of handwritten marks or classical proportions towards the modernist view of it as the endless manipulation of abstract elements. In modern typography, systematic relations across the body of the alphabet took precedence over the 'character' of individual letterforms (Lupton and Miller, 1996). For example, for the title of the Tamil film Ayyan, (Figure 1) the typeface has been manipulated such that the middle letter ' $y a a$ ' is altered to pop up over its two solidly flanking classical letters of ' $a a$ ' and ' $n a a$ '. The central letterform is rendered as an informal handwritten font as against the formal and classical appearance of its two neighboring letters, highlighting the aspect of being unique.

The title for the Tamil film Panchatanthiram also reflects the mix of hand-made and machine-made. While the type is derived from an existing font, the designer has cleverly integrated and digitally manipulated the English numerical ' 5 ' in red with the Tamil letter ' $i n j$ ' representative of the five male characters in the film. Having exploited the fact that the audience is equally familiar with the symbiotic relationship between the type and meaning of the word, the designer transfers meanings and associations from one to the other in the title itself.

In the early 1980s, type and image on a newspaper, brochure, or poster, were most often not integrated. The two firmly occupied their own spaces, often juxtaposed but rarely occupying the same space as evident in the Tamil films Uyarndha Ullam (Figure 2) and Samsaram Athu Minsaram. The pre-digital period demanded intense labor from artists and lettering artists, who spent several man-hours on one design. This became history with the advent of the computer and digital technology leading to simple, quick and stunning design solutions integrating type and imagery, changing the very aesthetics of Tamil cinema advertising as seen in films such as Hey Ram and Dasavataaram to name a few.

What sort of semiotic system is typography? The design of letterforms is a medium whose signifieds are not words but rather the alphabet. How has typography responded to its alphabetic signified? While Germany's Johann Gutenberg's fonts invented in c.1450 naturalistically simulated the variety and aura of handwriting, humanist designers at the turn of the century distanced the letter from calligraphy by constructing the Roman alphabet from the tools of geometry. The letterform was no longer thought of as a sequence of manual pen strokes, but as a conceptual ideal bound to no particular technology. This Platonic structure became typography's new signified (Lupton and Miller, 1996). This structure became typography's new signified. The alphabet is designed to represent speech. Writing is thus one language depicting another language, a set of visual signs representing vocal signs. This is echoed in the title design of the film Dum, Dum, Dum (Figure 3) which is clearly concept-driven and more than exemplifies the idea of typography standing in for the aural. The size 
hierarchy of the font alludes to the rising pitch of the melam (drum) that accompanies any Tamil Hindu muhurtham (auspicious wedding moment of marriage) ritual.

While English typography is structured and documented and its evolution can be traced with certainty, the Tamil script has not been studied as much-particularly from the point of view of design. Tamil is one of the classical languages of the world with a literary history of more than two millennia beginning with the Sangam age (300 BC - 200 AD). (Udaya Kumar, 2010) Originating from an ancient form related to the Devanagiri script, (Udaya Kumar, 2010) Tamil was written on palm leaves using a metal stylus. Charcoal powder was dusted over these leaves to make the writing visible. The high frequency of rounded shapes of the characters in this script is related to the manner of writing with the stylus (de Mello Vargas, 2007). Considered one of the oldest mediums of writing in ancient India, the precise origin and history of palm leaf writing are unknown but the practice is believed to have existed since the Sangam period (Udaya Kumar, 2010).

The Tamil script similar to most Indian languages, constantly encountered problems of adaptation when new technologies were implemented, especially amongst printers and computer users. However, more recently the realization of a functional typesetting scheme allied to the resources offered by OpenType assures a proper digital adaptation (de Mello Vargas, 2007).

The title design for the film Kannathil Muthamittal (Figure 4) made a radical reduction of the Tamil script to generate letterforms out of right angles and arcs of a circle. The formal parameter of this almost avant-garde typeface suppressed the individuality of the letters by forcing attention to the system of typography as a whole. By shifting the emphasis from the individual letter to the overall series of characters, the designer unconsciously evolved 'structuralist typography' exchanging the fixed identity of the letter for the relational system of the font. This novelty of manipulating the Tamil script played a typographic game, rendering the title of the film as an image as much as a word. The white title emerged out of a soft glow-like effect, resulting in a three-dimensional appearance. In some instances, subject to design and readability requirements, the soft glow assumed a colored tone unlike the regular grey that was most often used in the publicity. The typeface Mutham developed from this title font exists today as a digital font available for mass reproduction.

Aayitha Ezhuthu is a daring departure that made an attempt to change the staid style of commercial cinema by combining mainstream Tamil film aesthetics with international cinematic sensibilities. The story about fate and relationships revolves around three characters - a gangster, a student leader/social activist, and an engineerwhose lives intertwine from an incident that occurs on the landmark Napier Bridge in Chennai city. From that dramatic intersection, the story moves back and forth in time, following three separate flashbacks that occasionally brush against each other. The concept of three conjoined narratives and the fact that Aayitha Ezhuthu is the last letter of the Tamil alphabet appearing as vertices of a triangle, is distinctly developed in its title design. A typographic experimentation, it can be termed postmodern, having tested the structuralist limits of the letterform. The classical past has been displaced, yielding infinite variations.

According to art theorist and early architect of contemporary analytic aesthetics Clive Bell, aesthetic experience varies from everyday experience, and significant form is rooted in properties tangential, if anything, to everyday life. He describes it as arising from "relations and combinations of lines and colors, ... lines and colors combined in a particular way, certain forms and relations of forms [that] stir our aesthetic emotions" (Carol Gould, 1998, p. 252). For Bell the aesthetic nature and value are detached from its cultural, historical and personal origins; significant form is internal to the work, and as he observes, no historical, biographical, or psychological knowledge from life is needed to grasp this aesthetic property. One may see lines, colors and shapes visually but must focus on them and their relations in order to have an intuitive grasp of significant form. Significant form does in an important sense, signify. As Bell explains in his metaphysical hypothesis, it represents some ultimate reality. In some instances, he seems to mean by reality 'a visual reality stripped of interpretation, a reality that evokes the aesthetic response in the artist, who represents it for the audience' (Carol Gould, 1998, pp. 252-253). 
Susanne Langer in two books, Philosophy in a New Key (1942) and Feeling and Form (1953) further developed Bell's concept of significant form. By drawing interest to the way in which aesthetic forms are significant, she discards the idea of a particular aesthetic emotion debating that the forms of art somehow relate to formal features of human emotions in general. Further unlike language, which enjoys the aspect of being able to communicate precise meaning, Langer believes forms 'symbolize' feeling and are expressive of 'the life of feeling' in an articulate and organized way. In the belief that art conveys certain features of human feelings in a certain way, Langer's theory is a type of expressionism (Sheppard, 1987).

Much like a work of art, advertising for Tamil cinema has the peculiar property of conveying the aesthetic emotion, and it does this by virtue of having 'significant form'. The design for a particular Aayitha Ezhuthu poster with just the representation of the three black vertices, though geographically distanced, belong to Bell's formalism or Langer's expressionism, the key to which are their distinctive ideas of significant form as the defining element of an artwork or aesthetic object. By positioning the three 'painted' black dots on a stark orange background set within an irregular black border, the designer has tapped on the combination of the image and its implied benefits. The seemingly abstract dots do not merely represent 'aayitha ezhuthu' or the final word of the Tamil script, but are symbolically representative of the three protagonists.

The type used for the title Aayitha Ezhuthu is positioned on a seemingly hazy shadow, much like that of Kannathil Muthamittal and involves a combination of cinematic techniques such as harsh contrasts of light and shadow and complex camera movements highlighting the action-oriented theme of the film. Further, the designer has used compositional complexity, the layering of signs and pun in the meaning of the title and the decoding of the film's narrative. He has explored deconstructive graphics, and provokes the viewer into becoming an active participant in the construction of the message. Two of the three vertices are subtly incorporated in the Tamil version of the title, forcing the spectator to deconstruct 'hidden' meanings.

Ghajini is an action movie, propped up by romance and is based on a very interesting premise. The protagonist has been afflicted with short-term memory loss ever since he was hit on the head with an iron bar while trying to save his girlfriend. Except for this one incident that he clearly remembers, his memory span is limited. His daily existence is guided by clues he leaves himself through notes, photographs and self-inflicted tattoos. These pointers are meant to lead him to killing the villain. For the title design of Ghajini, the designer has drawn reference from the hand-made, particularly from graffiti art, rather than imitating traditional or classical typographic forms thereby reflecting the underlying focus of the film. The letterforms appear to be a scrawl referencing imperfection and irregularity derived from his reminder notes.

In the Tamil cinema industry, the use of themes allows films to be marketed in ways which inform potential audiences about the film, since posters, hoardings and other publicity ephemera contain coded signs which cue genre expectations. In most instances, the typographic design of the titles of Tamil films is indicative of the genre such as mythological, historical, patriotic, romantic or action oriented. Cultural association in terms of stylistic format is high key in the title design for the film Marudhanayagam (Figure 5). The Tamil typeface radically departs from the regular usage of the Tamil script to mimic Arabic calligraphy, making the typography expressive of the film and its genre, which revolves around the protagonist being a Muslim king. Similarly the title design for Viswaroopam (Figure 6) offers a visual narrative of the film with its unique mix of modernism and individualism. Reflective of the narrative centred around an Islamic protagonist posed as a Hindu Kathak dancer caught in the web of the Al-Qaeda war against the Americans, the title of the film is stylized to resemble Arabic script distinctly different from that of Marudhanayagam. The design therefore performs a potentially expressive function in the articulation of meaning, crowning it with a specific cultural and religious reference.

On a concluding note it can be reiterated that digital technology that is man-made has come to the creator's aid in simplifying the clutter of the so-called design space in Tamil movie promotionals-in reducing the component elements and articulating maximum message with minimum means. This paper has highlighted how 
typography has come to have a life of its own and has a specific role to play instead of merely being suggestive of formal grandeur alone. These promotionals clearly signify that the Tamil filmgoer has been sensitized to a design aesthetic that is revealing of his sophisticated visual comprehension.

Chennai, which enjoys a happy balance between the traditional and the modern, exhibits the same in its publicity of Tamil films as well. Outdoor advertising for Tamil cinema, while being hinged to the cinema that it publicizes, has emerged as a site of negotiation for recent and advancing technologies and socio-cultural trends. The pivot of this negotiation is the creativity of the designer augmented by the available technological devices that has brought in slick and sophisticated advertising solutions for the Tamil film industry. While elevating the Tamil film viewers' sense of reception, the audience is now receiving visual stimuli akin to the global viewer.

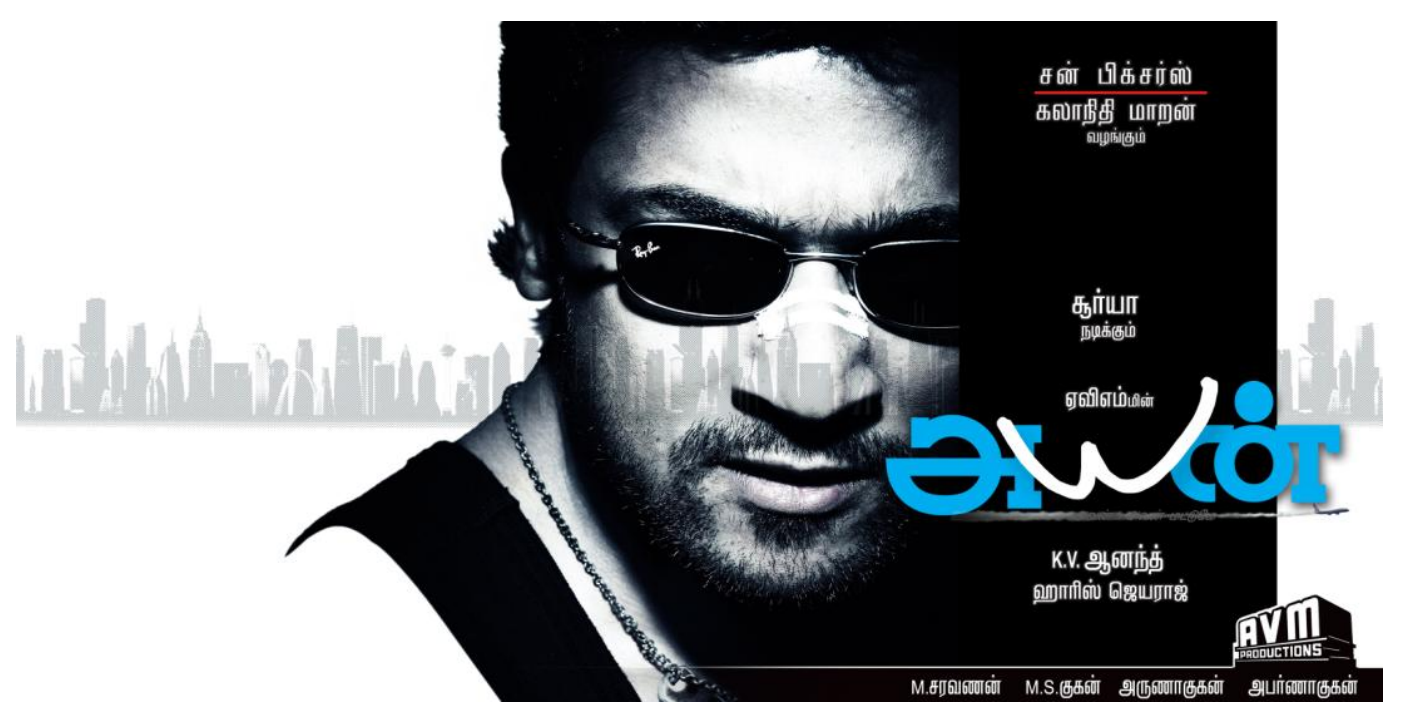

Figure 1

Ayyan (Unique), Hoarding, 2009

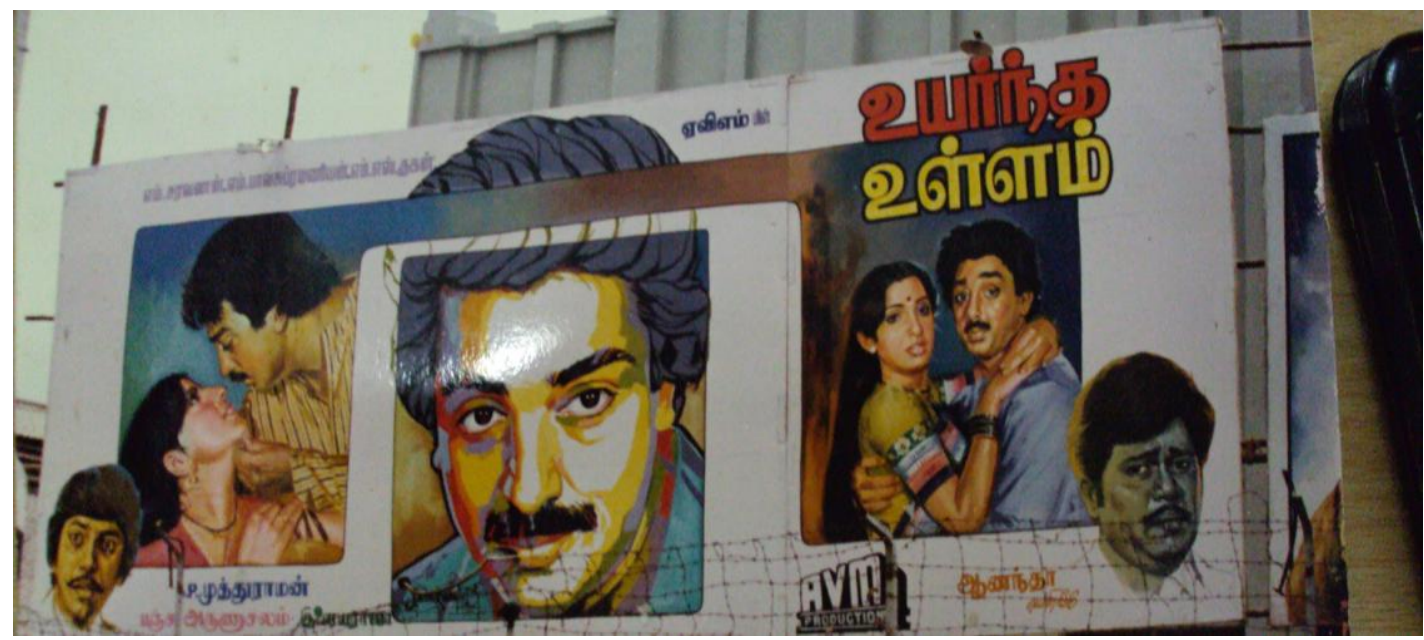

Figure 2

Uyarndha Ullam (Noble Heart) Hoarding, 1985 


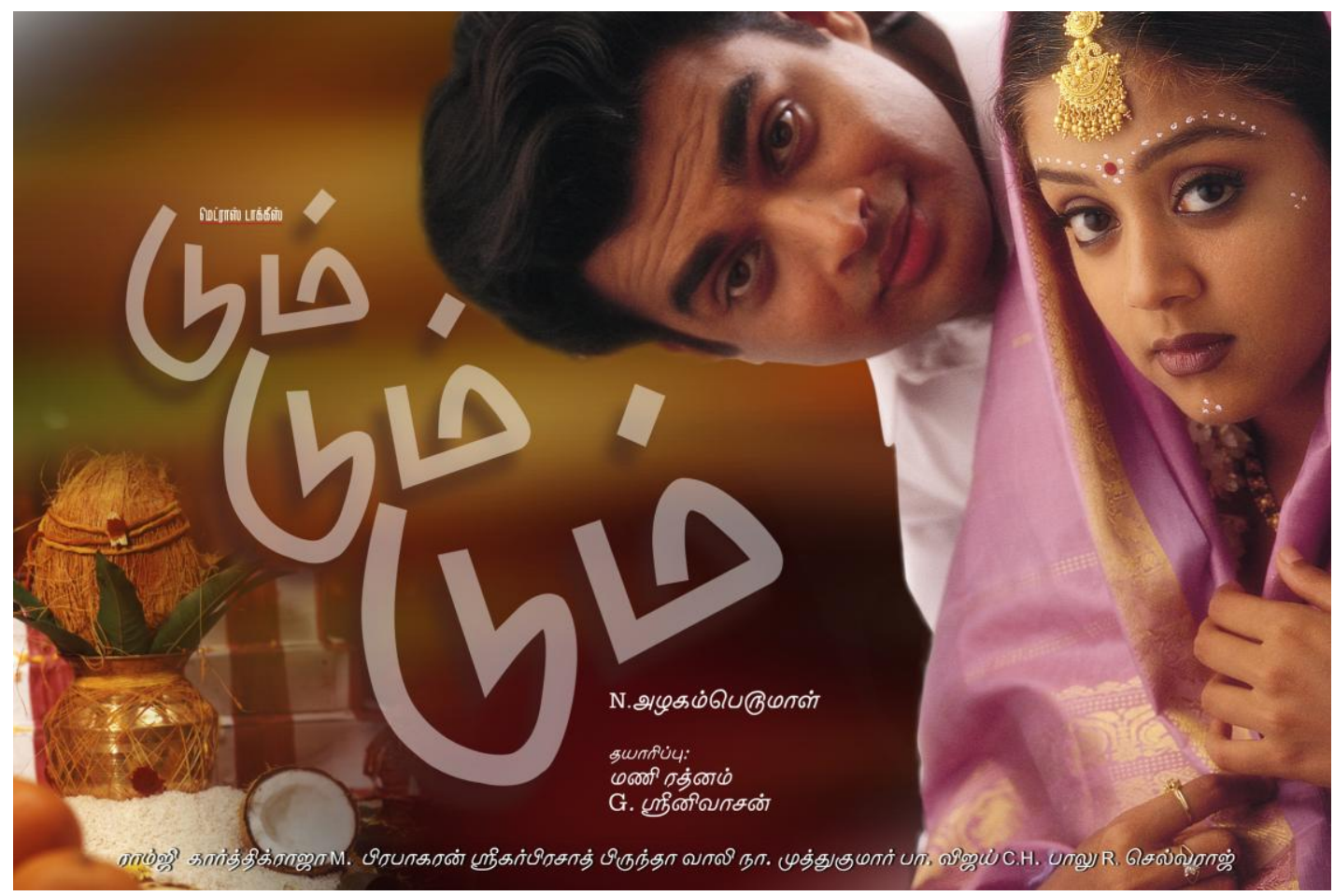

Figure 3

Dum, Dum, Dum, Poster, 2001

A M A N I R A T N A M FIL M

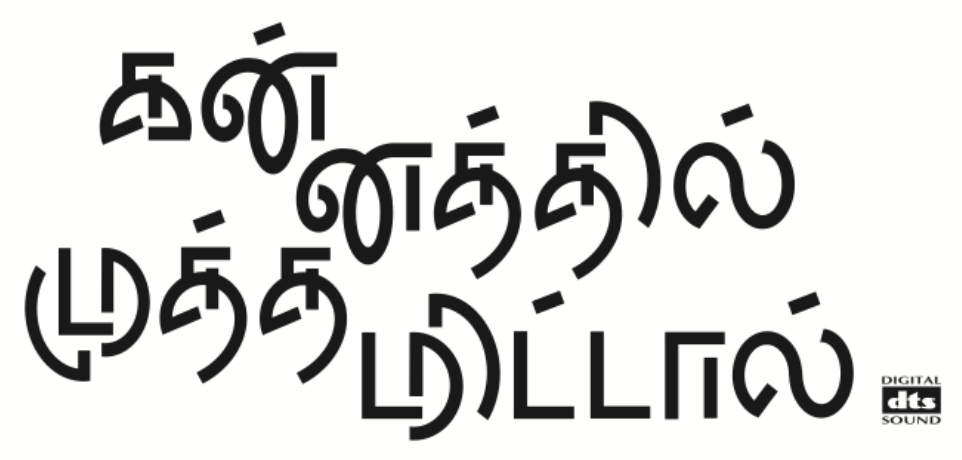

Figure 4

Kannathil Muthamittal (A Peck on the Cheek) Title Design, 2009 


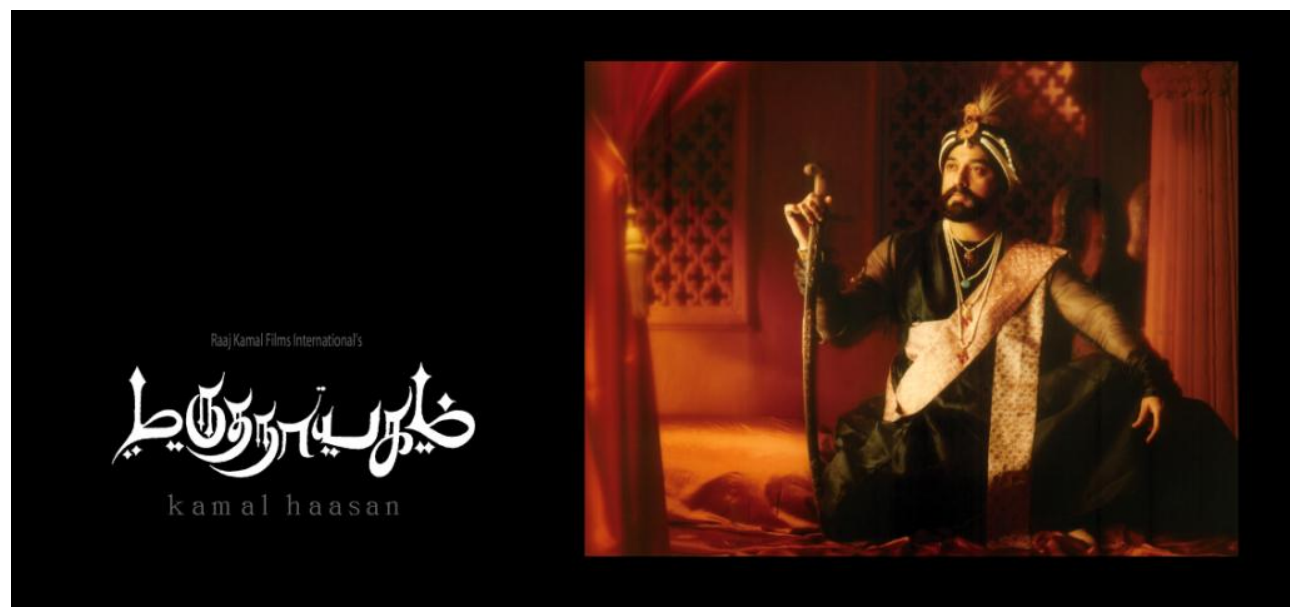

Figure $5 \quad$ Marudhanayagam (yet to be released)

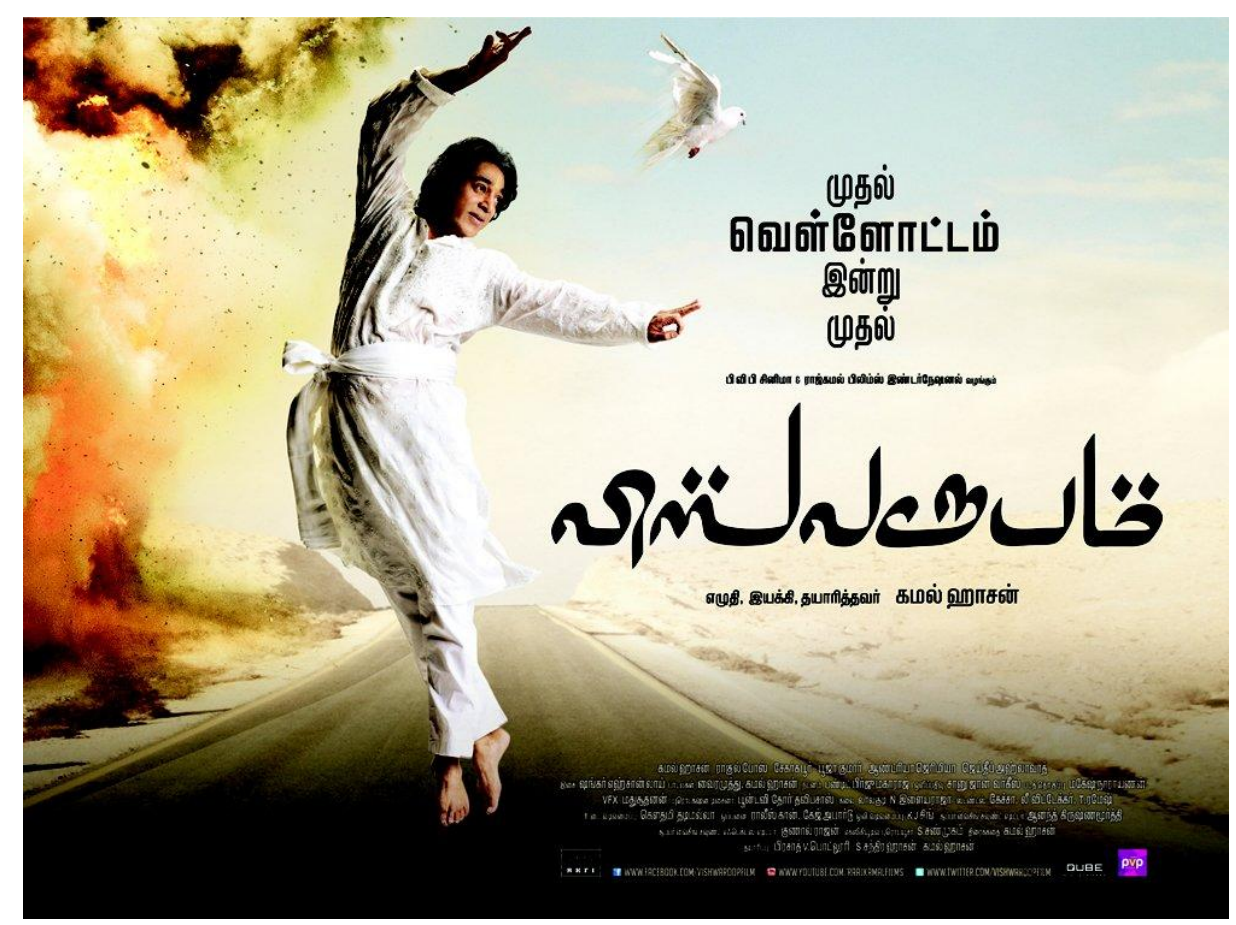

Figure $6 \quad$ Viswaroopam (The Cosmic Form), Poster, 2013

\section{References}

Benjamin W. 1936, The Work of Art in the Age of Mechanical Reproduction, (London, U.K.: Penguin Books).

Carol Gould, B., 1998, Arthur Clive Howard (1881-1964). In: Encyclopedia of Aesthetics, edited by M. Kelly. Vol. 1 (Oxford, U.K.: Oxford University Press), p. 252.

de Mello Vargas, F., 2007, Evolution of Tamil typedesign: origins and development. M.A. dissertation, Department of Typography and Graphic Communication, (Reading, U.K.: University of Reading).

Lister, M., 1995, Photographic Image in Digital Culture (London, U.K.: Routledge).

Lupton, E. and Miller, A., 1996, Design Writing Research: Writing on Graphic Design (London, U.K.: Phaidon).

Murphie, A., and Potts, J., 2003, Culture and Technology (New York: Palgrave Macmillan). 
Sheppard, A., 1987, Aesthetics: An Introduction to Philosophy of Art (Oxford, U.K.: Oxford University Press). Udaya Kumar D., 2010, Transformation of Tamil letterforms from palm leaf manuscripts to early letterpress printing. Ph.D Dissertation, Industrial Design Centre, Indian Institute of Technology, Mumbai. www.idc.iitb.ac.in/students/phd/D_Udaya.htm. 